\title{
Design and Implementation of MES for Discrete Manufacturing Workshop of an Aerospace Enterprise
}

\author{
Zhang Guangshen", Yu Changyong, Wang Yan \\ Information and Software Development Center, Beijing Institute of Aerospace Control Devices, Beijing, China
}

\section{Email address:}

Fanzgs@163.com (Zhang Guangshen)

${ }^{*}$ Corresponding author

\section{To cite this article:}

Zhang Guangshen, Yu Changyong, Wang Yan. Design and Implementation of MES for Discrete Manufacturing Workshop of an Aerospace Enterprise. Science Discovery. Vol. 9, No. 2, 2021, pp. 68-73. doi: 10.11648/j.sd.20210902.18

Received: March 17,2021; Accepted: April 22, 2021; Published: April 26, 2021

\begin{abstract}
MES belongs to the production execution system of manufacturing enterprises. When applied to the production process, MES can effectively promote the construction of enterprise information and improve the market competitiveness of enterprises. This paper takes the discrete manufacturing workshop of an aerospace company as the research object, aiming at the characteristics of its multi-variety, small batch, and discrete production, combining the current production management status of the workshop and the functional requirements of information business, guided by advanced concepts such as lean production Thought, proposed the MES system architecture design plan, built an aerospace enterprise discrete manufacturing workshop MES. The system mainly includes process management, material management, equipment management, plan management, special process management, quality management, data collection and other functions. The system opens up the production process data link, realizes process visualization, material life cycle management, and fine production equipment management, production planning process control, special process monitoring and tracking, process quality data analysis, and automatic collection of generated equipment data. And the system have formed a digital analysis platform suitable for aerospace discrete manufacturing workshops, solving the actual production management problems of aerospace enterprises' discrete manufacturing workshops and improving It improves the information management level and production efficiency of the workshop, and provides ideas for the leap-forward improvement and development of the production and manufacturing capacity of the enterprise.
\end{abstract}

Keywords: Aerospace Enterprise, Discrete Manufacturing, MES

\section{某航天企业离散制造车间MES设计与实现}

张广申", 于畅泳, 王岩

信息化与软件开发中心, 北京航天控制仪器研究所, 北京, 中国

\section{邮箱}

Fanzgs@163.com(张广申)

摘要：MES属于制造企业生产执行系统，把其应用到生产过程中，可以有效促进企业信息化建设，提高企业的市场竞 争能力。本文以某航天企业离散制造车间为研究对象, 针对其多品种、小批量、离散化生产的特点, 在结合该车间生 产管理现状和信息化业务功能需求的基础上, 以精益生产等先进理念为指导思想, 提出了MES系统架构设计方案, 并 构建了某航天企业离散制造车间MES。系统主要包括工艺管理、物料管理、设备管理、计划管理、特殊过程管理、质 量管理、数据采集等功能, 系统打通了生产过程数据链路, 实现了工艺可视化、物料全生命周期管理、生产设备精细 化管理、生产计划过程管控、特殊过程监测跟踪、过程质量数据分析和生成设备数据自动采集, 形成了适用于航天离 
散制造车间的数字化分析平台, 解决了航天企业离散制造车间生产管理实际问题, 提升了车间的信息化管理水平和生 产效率，为企业生产制造能力跨越式的提升和发展提供了思路。

关键词：航天企业，离散制造，MES

\section{1. 引言}

制造业是我国国民经济的支柱产业，随着“中国制造 2025”战略的推进，很多企业已经逐步开展生产经营信息 化建设, 实施信息化与自动化深度融合 $[1,2]$ 。

军工制造企业尤其是航天企业承担着国家重器制造 的责任, 在满足生产质量的前提下, 如何提高航天企业的 信息化管理水平和生产效率成为了亟待解决的问题 [3]。越 来越多的航天军工企业已经深刻地意识到, 大力发展和应 用信息技术，用先进的技术管理手段实现生产过程管控的 数字化, 通过实施具有精益生产思想的、适合军工企业特 点的数字化车间管理系统, 即构建以“精益生产、协同制 造”为特色的MES, 可很好地解决上述问题 [4]。

\section{MES概述}

制造执行系统 (Manufacturing execution system, MES) 是一套应用于制造业车间生产的信息化管理系统[5]。该系 统概念最早形成于 20 世纪 80 年代后期, 欧美发达国家率先 发展和应用, 进入 21 世纪后MES才引起国内企业的重视并 逐步得到发展与应用 [6]。美国先进制造研究机构 (Advanced Manufacturing Research, AMR) 将MES 定义为 “位于上层的计划管理系统与底层的工业控制之间的, 面 向车间层的管理系统”[7], 主要包括资源管理、详细计划、 排产调度、文档管理、数据采集、人力资源管理、质量管 理、过程管理、设备维护管理、生产跟踪和性能分析 11 个功能模块。MES的主要作用是为企业生产过程提供帮助, 将生产计划与现场管理有机结合, 实现人员、设备、物料 等生产资源的合理配置, 提高资源利用率。

由于MES与生产工艺及管理流程密切相关, 不同行业 的生产工艺和管理模式差异较大, 因此MES具有鲜明的行 业特色[8]。在实际MES建设过程中, 根据企业实际业务需 求以及MES应用的深度和广度, 通常只建设其中几个功能 模块。

\section{3. 车间生产管理现状与需求分析}

\section{1. 生产管理现状}

某航天企业离散制造车间为研究所下属制造单位, 承 担着研究所产品的生产任务, 生产产品主要为单机, 业务 范围包括装配和调试两部分, 不仅具有普通制造业的普适 性, 也有军工制造业的独特性, 其生产体系如图1所示。 车间目前生产管理现状如下:

(1) 多品种、小批量、离散化生产
生产种类较多，不同种类产品的制造批量较小。整个 生产执行过程分布在若干分散场地, 车间生产采用典型的 离散型生产组织模式。

(2) 科研与生产并重, 临时任务较多

承担多种型号产品试制和批产任务, 造成试制和批产 任务交叉进行, 生产工艺和制造过程调整频繁。车间时常 接到一些临时紧急任务, 此类插单任务原本不在生产计划 中，给车间生产计划制定和调整带来极大挑战。最终导致 计划的精确性较差, 编制效果与调整的效率较差, 容易造 成资源利用不充分、不能按期交货等问题[9]。

制造工艺复杂、产品质量要求高

车间产品均为航天军工产品, 航天军工企业除了具备 一般离散制造业的特点之外, 还具有结构复杂、零部件数 量多、周期长等特点 [10], 制造难度较大, 产品规格和质 量要求较高。

（4）人员技能水平对产品合格率影响较大

车间生产作业计划和调度主要依赖人工经验来完成, 由于车间产品工序复杂, 从生产成本和生产时间考虑, 大 部分工序采用手工生产, 只有部分工序采用自动化生产, 因此人员技能水平对产品合格率影响较大。

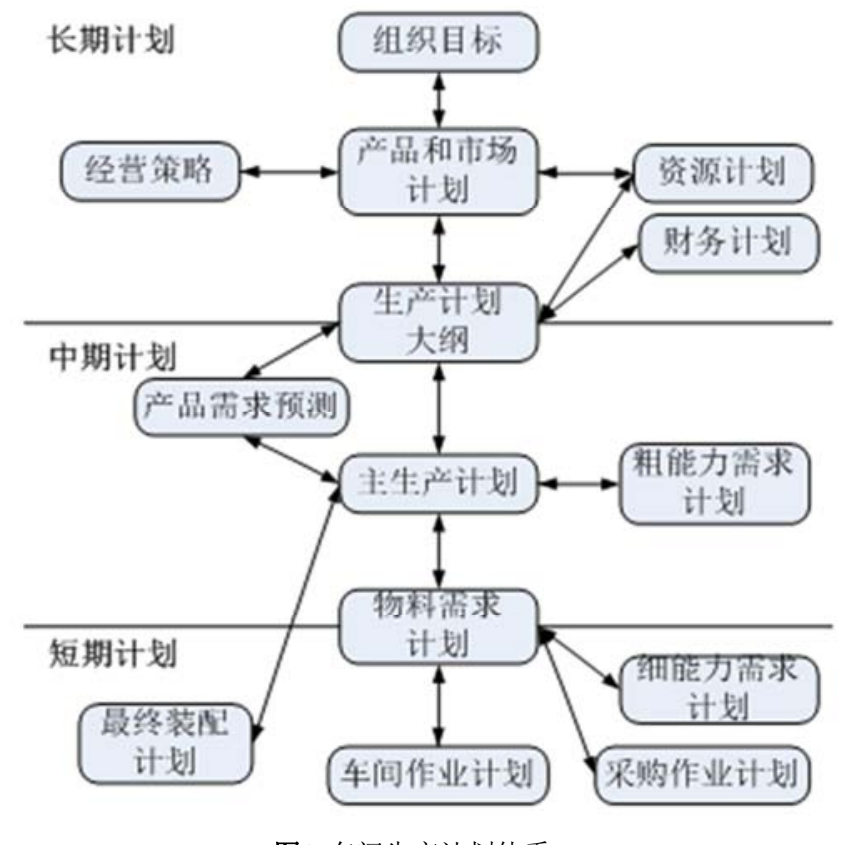

图1 车间生产计划体系。

\section{2. 生产管理需求分析}

通过对车间生产管理现状进行分析和业务需求调研, 归纳出车间生产管理中出现的主要痛点, 具体如下。

（1）“信息孤岛”现象严重 
车间目前没有较为完整的生产管理系统, 缺乏高效的 信息汇总和传递手段，车间人员、设备、物料等生产资源 信息不够精确、清晰, 生产现场信息主要依靠电话或者电 子台账获得, 信息传递速度慢, 问题不能及时反馈和快速 解决, 手工管理工作量大, 管理效率低下。

（2）生产计划制定困难

产品品种多、批量小、插单严重, 影响因素较多, 计 划编制困难[11]。生产计划的制定依赖于调度人员, 主要 依靠生产经验, 缺乏有效的科学计划排程体系支撑, 不能 高效、科学的排产。

(3) 生产过程跟踪监控困难

车间生产过程跟踪监控主要通过人工方式进行, 缺乏 对当前生产状态及各种物料、设备等资源的动态掌握, 无 法进行生产数据全流程追踪, 无法实时准确把握生产现场 状况, 影响作业计划的执行。

\section{MES系统设计}

根据车间生产管理现状与业务需求, 以分配订单生产 执行为主线, 利用业务流程内含的信息流整合制造过程中 物流、工艺流所涉及的人、机、物、法、环等诸多生产要 素, 依据生产制造特点和信息流自身的规律并借助信息技 术重新设计信息流的结构, 实现制造过程信息的高效收集、 分析、决策和反馈, 形成闭环的管理过程。最终达到企业 对生产制造的协调、规范和考评, 推动企业对各项业务的 控制、改善和提升的目标。

\subsection{MES系统架构}

如图2所示, 本文所设计MES主要功能模块有工艺管 理、物料管理、设备管理、计划管理、质量管理、数据采 集、特殊过程管理等, 系统功能模块如图3所示。

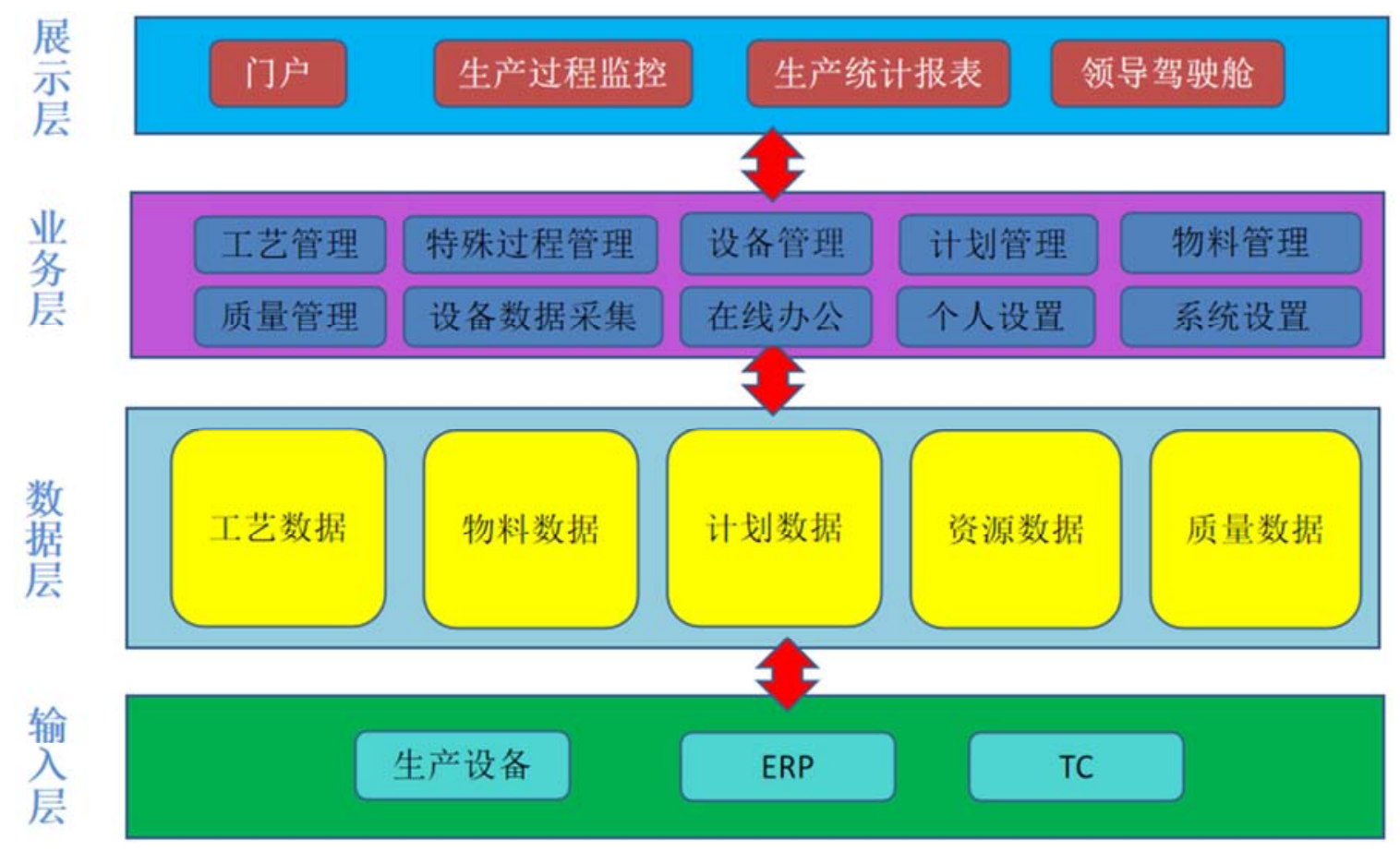

图2 MES系统架构图。

\section{2. 工艺管理}

在制造企业中, 工艺设计作为联系产品设计与制造的 中枢, 是整个制造过程中的重要环节, 对产品制造具有极 为重要的影响 [12]。

车间产品工艺工序复杂, 生产环节多, 大部分工序手 工生产, 因此需要严格的工艺管理和工艺指导, 工艺管理 模块主要进行产品结构、工艺文件、工艺可视化、工时定 额等管理, 有助于车间工艺规程规范化, 同时通过系统线 上工艺指导, 可以有效提高操作人员工艺水平, 提高产品 合格率。

\section{3. 物料管理}

车间库房各类零组件、原材料、辅料种类繁多, 物料 管理主要进行物料验收、出入库、库存盘点等工作。物料
与产品 BOM关联, 通过生产过程数据建模挖掘分析产品 生产时的最优齐套配料方案并固化到生产过程质量控制 及物料数据管理中。可对将来的库存情况进行预测, 增加 零组件、原材料、辅料库存管理的可控度, 在保障供应的 情况下降低库存量, 进而降低库存成本, 缩短生产准备时 间。

\section{4. 设备管理}

设备管理是生产过程中的关键内容, 建设设备管理模 块, 进行有效统一地管理和维护车间生产制造过程中所需 使用到的各种设备, 详细记录设备全生命周期中的管理和 维护动作, 其涉及范围主要包括: 设备台账、维修送检、 设备状态管理等。该模块会对每台设备分配唯一编号, 该 编号贯穿设备的整个生命周期。同时, 借助生产过程数据 
能够分析设备的生产运行情况和故障趋势, 为改进设备和 优化设备生产方案提供支持。

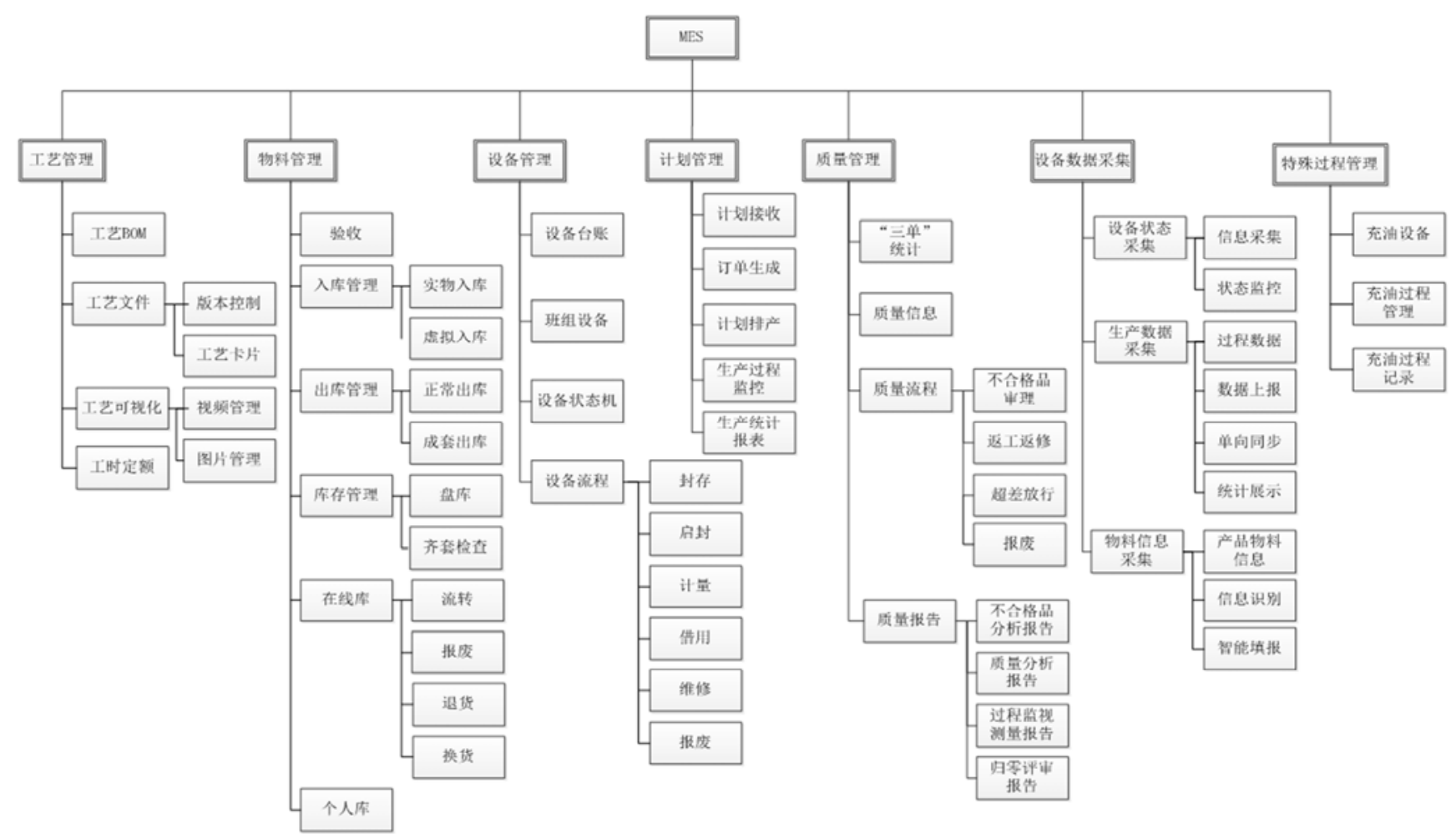

图3 MES功能模块图。

\section{5. 计划管理}

生产计划管理是MES的核心模块, 实现了生产业务流 的闭环。该模块支持新增生产计划及接收上层系统推送过

来的计划, 系统将主计划分解为子计划, 根据产品工 艺和物料数据进行生产计划的排程。在计划排程过程中, 综合考虑整条生产线的物流及约束情况、工序间的依赖关 系、企业内外资源及能力约束，通过优化模型与各种优化 算法, 提高排程效率[13]。排程后的生产计划经审核通过 后对所需的物料进行齐套分析和配套出库，下发到车间进 行生产, 并对计划执行情况进行过程管控, 支撑智能管控 和生产决策, 降低对经验人员的依赖。

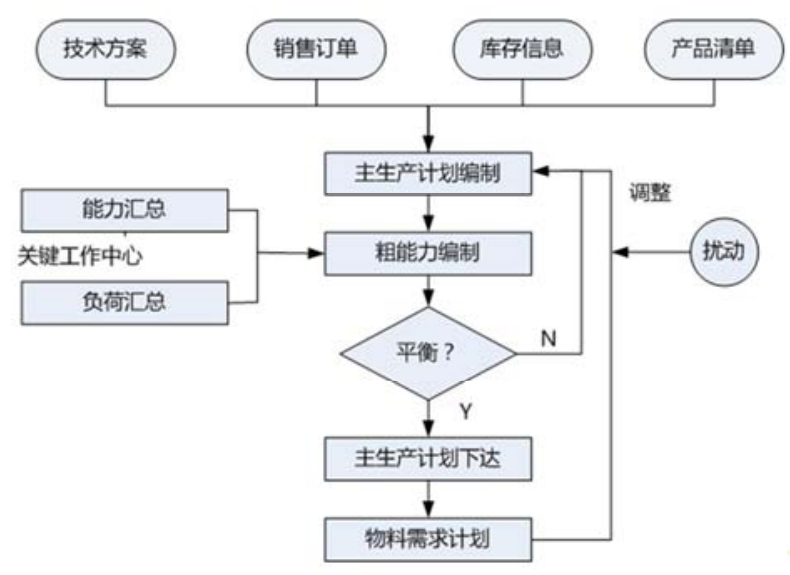

图4 计划编制过程。

\section{6. 质量管理}

产品质量管理是企业的生命线，涉及产品设计、制造 等多个方面, 其中生产过程是产品质量管理最为繁杂、影 响因素最多的过程。

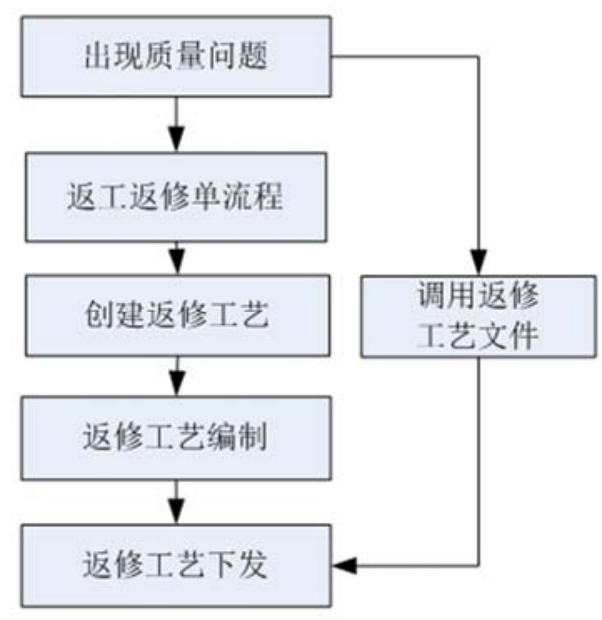

图5 返工返修流程。

本系统通过检验、分析、控制 3 个环节，实现对生产 全过程的质量信息的管理和控制, 将生产线中工序与工步 的填报校验功能和装配流程的质量管理相结合 [14]。首先, 根据检验工位出具的检验数据与工艺要求进行对比, 出现 偏离需形成不合格品审理单, 并驱动不合格品审理流程。 不合格品未经审理不能继续加工或转入下道工序。通过评 
审后, 需要明确审理结论。审理结论为返工 (返修) 时, 生成返工 (返修) 通知, 由工艺员编写返工 (返修) 临时 工艺文件后进行返工（返修）生产流程。审理结论为报废 时, 生成报废记录。审理结论为超差放行时, 生产超差放 行记录。返修、废止的审理结论都需要经过严格的审批流 程。系统具备记录产品质量状况的功能, 对产品的全部工 序记录其质量状况, 做产品质量追溯的同时支持统计、分 析, 形成不合格品分析报表。

\section{7. 设备数据采集}

为强化MES对生产车间的数据采集和监控能力, 系统 通过分布式数控(Distributed Nu-merical Control, DNC)、 条码枪和刷卡器等实现车间生产信息的采集。

系统可以通过RFID读写器、手持PDA、传感器等多种 方式采集设备运行状况和生产过程数据等信息, 通过汶览 器、电子看板等方式实现数据的综合展示 $[15,16]$ 。通过对 车间设备状态采集与监控、生产数据采集、生产进度监控、 物料信息采集等功能。可以消除上层管理系统与底层车间 的信息鸿沟, 使工作人员全面掌握车间现场的实时生产情 况。搭建生产管理层和底层控制系统之间的信息桥梁, 为 生产管理决策和调度提供重要的数据支持。同时对生产现 场各个生产要素进行状态和行为监控并且对异常情况进行 报警, 将采集到的生产要素和生产过程中的各类数据, 存 储到数据库中, 可以作为生产数据挖掘与分析的依据。

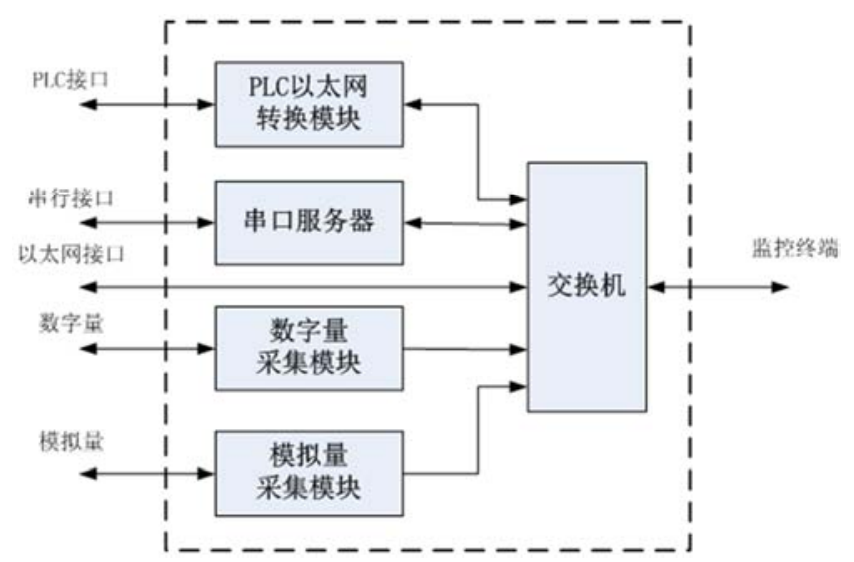

图6 数据采集终端结构。

\section{8. 特殊过程管理}

航天军工产品生产过程复杂, 其中部分工序为关键工 序, 在生产过程中需格外关注, 因此系统根据实际生产需 求建设特殊过程管理模块。

本系统的特殊过程管理以产品充油过程为例, 通过实 时监控产品充油过程, 及时反馈和快速解决产品充油过程 中遇到的问题, 确保产品生产过程中的特殊过程不出问题, 为产品生产提供了有力保障。

\section{MES系统实现}

系统基于 $\mathrm{J} 2 \mathrm{EE}$ 技术架构和关系数据库搭建, 采用 $\mathrm{B} / \mathrm{S}$ 模式, 采用面向对象的思想和方法进行设计与开发。系统
使用Spring Framework框架, Spring MVC为模型视图控制 器, MyBatis为数据访问层, Apache Shiro为权限控制层, Ehcahe 为数据缓存控制, Activiti为工作流引擎, 使用 Maven做项目管理，前端使用CSS、JQuery和Bootstrap技 术, 后台使用Java EE技术, 数据库采用MySQL数据库。具 有良好的可配置性、扩展性与可集成性, 不同企业的工艺 流程与管理需求可以通过现场的定义在系统中实现, 系统 技术架构如图7所示。

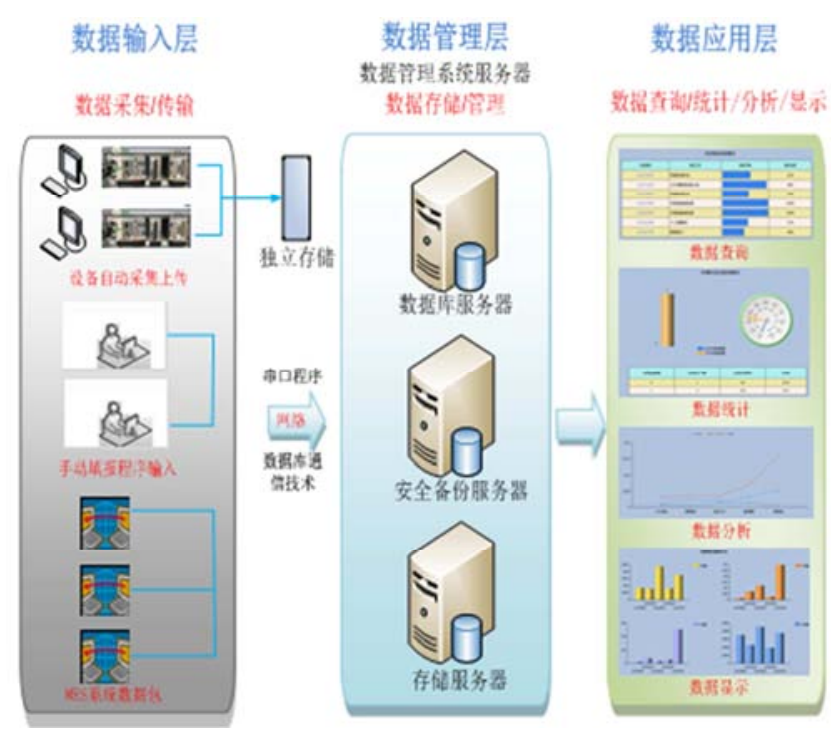

图7 系统技术架构。

系统主要界面如图所示:

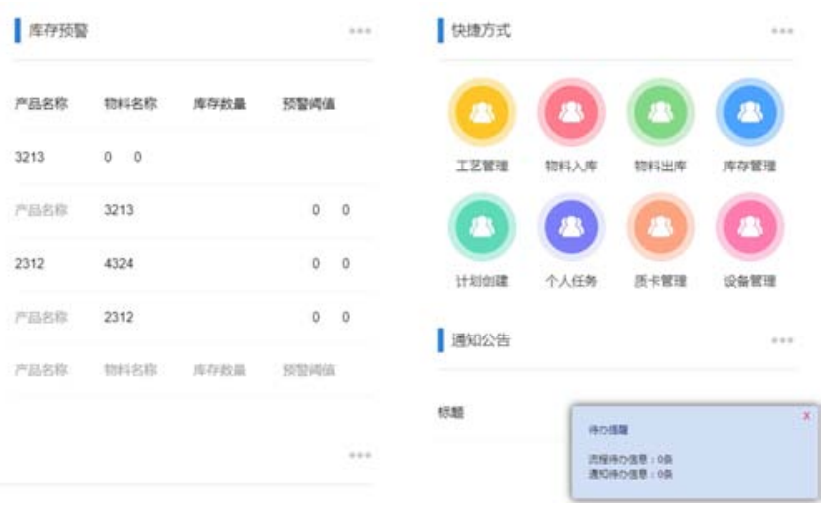

图8 系统首页。

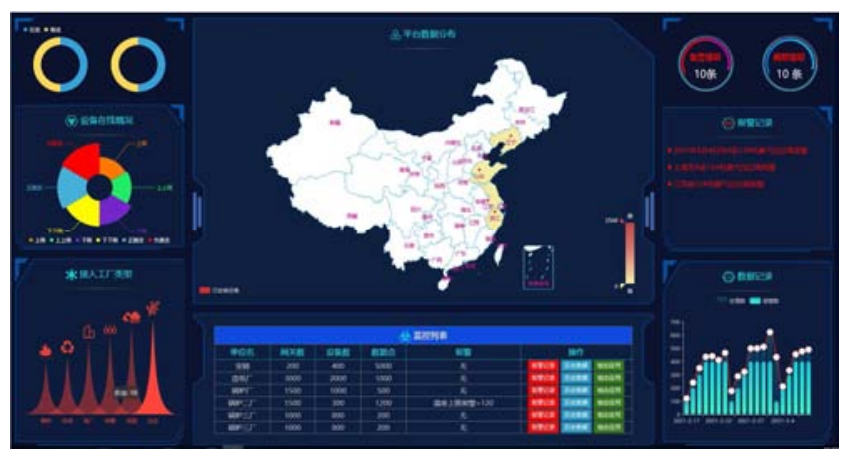

图9 系统统计分析界面。 


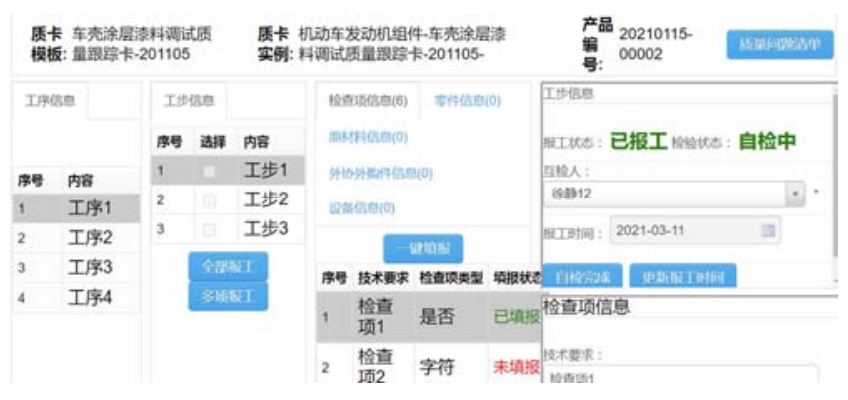

图10 质卡填报界面。

\section{6. 结论}

本文以某航天企业离散制造车间为研究对象, 设计并 实现了MES系统, 系统打通了生产过程数据链路, 实现了 工艺可视化、物料全生命周期管理、生产计划过程管控、 过程质量数据采集、设备精细化管理、设备数据采集、特 殊过程关注等功能, 形成了适用于航天企业离散制造车间 装配、调试、数据管控与分析的数字化平台, 实现了信息 快速收集、便捷查询、高效利用、综合分析, 同时规范生 产部门生产流程和员工操作。

该系统的实施应用, 优化了车间生产管理方法, 减 少了产品生产过程中人为因素的影响, 解决了生产现场 信息传递滞后问题, 实现了生产过程管控数字化, 对解 决目前生产现场效率低, 交付周期长等问题具有一定的 指导作用。

\section{参考文献}

[1] 李清.制造执行系统[M].北京:中国电机出版社,2007.

[2] 谭芳.MES环境下的企业质量管理方法探究[J]. 装备制造技 术.2020,5(5):265-269.
[3] 陆国强.MES 系统实现精益生产 [J]. 新技术新工 艺.2017(7):2-3.

[4] 谢光文, 马淑玲,朱铎先. 军工行业MES系统的 5 大关键技术 [J].新技术新工艺.2012（7）:14-18.

[5] 郑唯唯, 梁俊俊.多品种、小批量产品过程质量集成控制研 究[J].企业管理与信息化,2007(7):19-21+25.

[6] 姚涛.船舶军工制造企业MES应用研究[J].现代制造工程, 2017(3):31-37.

[7] 刘艳.制造执行系统 (MES) 在航天电子企业中的应用研究 [D].西安:西北大学,2010.

[8] 梁永宏,张吴,黄康培,谢霞.制药行业MES产品的设计与实现 [J].机电工程技术,2020,49(07):166-168.

[9] 赵声吨.智能制造及其核心信息设备的研究进展及趋势[J]. 机械科学与技术.2017 36(1).

[10] 费一正.面向离散制造的协同制造执行系统及其关键技术 研究[D].南京:南京理工大学,2011.

[11] 孟翔.某航天产品装配生产MES研究[D].南京:南京航天航 空大学,2014

[12] 钱芳, 李小虎.面向APS 系统的MES系统改造方案及实现[J]. 冶金动力.2019(10),104-106

[13] 李丽,武照云,宋吴举等.基于移动端APP的机械加工工艺卡 片管理系统[J].机械设计与制造,2021(01):301-304.

[14] 任耀华.面向光伏电池数字化车间的系统设计 $[\mathrm{J}]$. 电子工业 专用设备,2020,49(04):50-53.

[15] 袁敏娟, 曾云翔. 基于RFID的实时缝制数据采集及生产管 理系统[J].自动化技术与应用,2020(02):150-153.

[16] 郭苗,孟邹清.浅析煤化工企业智能工厂现场数据采集[J].中 国仪器仪表,2018(07):26-28. 